Article

\title{
Changbai Mountain Ginseng (Panax ginseng C.A. Mey) Extract Supplementation Improves Exercise Performance and Energy Utilization and Decreases Fatigue-Associated Parameters in Mice
}

\author{
Guo-Dong Ma ${ }^{1}$, Chun-Hui Chiu ${ }^{2,+}$, Yi-Ju Hsu ${ }^{3,+}$, Chien-Wen Hou ${ }^{4}$, Yi-Ming Chen ${ }^{3, *}$ \\ and Chi-Chang Huang ${ }^{3, *}$ \\ 1 Sport Science College, Jilin Sport University, Changchun 130022, Jilin, China; magd2008@126.com \\ 2 Graduate Institute of Health Industry Technology, Research Center for Industry of Human Ecology and \\ Research Center for Chinese Herbal Medicine, College of Human Ecology, Chang Gung University of \\ Science and Technology, Taoyuan 33303, Taiwan; chchiu@mail.cgust.edu.tw \\ 3 Graduate Institute of Sports Science, National Taiwan Sport University, Taoyuan 33301, Taiwan; \\ 1041302@ntsu.edu.tw \\ 4 Laboratory of Exercise Biochemistry, Department of Sports Sciences, University of Taipei, Taipei 11153, \\ Taiwan; om65726@yahoo.com.tw \\ * Correspondence: 1021302@ntsu.edu.tw (Y.-M.C.); john5523@ntsu.edu.tw or d301090007@gmail.com (C.-C.H.); \\ Tel.: +886-3-328-3201 (ext. 2409) (Y.-M.C. \& C.-C.H.) \\ + These authors contributed equally to this work.
}

Academic Editors: Anusha Chaparala, Lorne Hofseth and Woo-Sik Jeong Received: 11 December 2016; Accepted: 3 February 2017; Published: 5 February 2017

\begin{abstract}
Changbai Mountain Ginseng (CMG, Panax ginseng C.A. Mey) is a traditional medicine commonly found in Northeast China and grows at elevations of $2000 \mathrm{~m}$ or higher in the Changbai Mountain Range. CMG, considered to be a "buried treasure medicine", is priced higher than other types of ginseng. However, few studies have demonstrated the effects of CMG supplementation on exercise performance, physical fatigue, and the biochemical profile. The major compound of CMG extract was characterized by electrospray ionization tandem mass spectrometry (HPLC-ESI-MS/MS). Male ICR mice were divided into 3 groups, the vehicle, CMG- $1 \mathrm{X}$ and CMG-5X groups $(n=8$ per group), and respectively administered 0,5 , or $25 \mathrm{mg} / \mathrm{kg} /$ day of CMG extract orally for four weeks. HPLC-ESI-MS/MS results showed that the major compound in CMG extract is ginsenoside Ro. CMG extract significantly increased muscle weight and relative muscle weight (\%). CMG extract supplementation dose-dependently increased grip strength $(p<0.0001)$ and endurance swimming time, decreased levels of serum lactate $(p<0.0001)$, ammonia $(p<0.0001)$, creatine kinase $(C K$, $p=0.0002)$, and blood urea nitrogen $(p<0.0001)$, and economized glucose levels $(p<0.0001)$ after acute exercise challenge. The glycogen in the gastrocnemius muscle was significantly increased with CMG extract treatment. Biochemical profile results showed that creatinine and triacylglycerol significantly decreased and total protein and glucose increased with CMG treatment. This is the first report that CMG extract supplementation increases muscle mass, improves exercise performance and energy utilization, and decreases fatigue-associated parameters in vivo. The major component of CMG extract is ginsenoside Ro, which could be a potential bioactive compound for use as an ergogenic aid ingredient by the food industry.
\end{abstract}

Keywords: ginsenoside; Ro; exercise performance; anti-fatigue; muscle mass 


\section{Introduction}

Changbai Mountain ginseng (Panax ginseng C.A. Mey, CMG) is a wild ginseng that grows in the Changbai Mountain Range in Jilin Province, the highest mountains $(2750 \mathrm{~m})$ in Northeastern China. CMG is harvested from the local forests and has been used as a traditional Chinese medicine (TCM) for more than a century. Recently, the Chinese government has developed large wild ginseng growing bases, where traditional Chinese herbs are produced, to meet the Good Agricultural Practices (GAP) standards and encourage the development of the pharmaceutical industry in Northern China [1]. In general, the two major species of ginseng are Asian (Panax ginseng) and American (Panax quinquefolius) [2]. In previous reports, a common mixture of active ingredients was demonstrated to be ginsensosides [3-5]. The benefits of ginseng are attributed to bioactive compounds, such as ginsenosides, volatile oils, polyphenols, flavonoids, polysaccharides, and vitamins [6-10]. In previous reports on physiological functions, ginseng exhibited anti-cancer [11], immunomodulatory [12], and antidiabetic [13] effects, and cardiovascular improvement [14] in delivering ginseng ginsenosides. Ginseng roots vary from different regions, and differences in environmental factors could influence the genotypes [15]. Thus, CMG could be more bioactive than ginseng grown in other locations. The aim of the current study was to evaluate the anti-fatigue activities of ginseng polysaccharides, as well as the neutral, acidic portions and ginseng polysaccharides of CMG [16]. However, there is a lack of research to support the anti-fatigue effects of the major compounds of CMG, which could improve exercise performance. In this study, we identified the major compound of CMG extract and evaluated the ergogenic, anti-fatigue, and health promotion effects of CMG supplementation using our previously established in vivo platform $[17,18]$.

\section{Results}

\subsection{Characterized the Major Compound of CMG Extract by HPLC-ESI-MS/MS}

CMG was systematically characterized by electrospray ionization tandem mass spectrometry (HPLC-ESI-MS/MSn). Figure 1a shows the ginsenoside standard chromatogram (Rc, Rb2, Rd, Rg2, $\mathrm{Rb1}$, Rf, Re, Rg1); no ginsenoside Rc, Rb2, Rd, Rg2, Rb1, Rf, Re, Rg1 were found in the CMG extract (Figure 1b). The major compound was found at a $44.2 \mathrm{~min}$ retention time. The major parent ion was detected and processed for multiple fragment analysis. As shown in Figure 2a, the MS1 parent ions were identified as $955 \mathrm{~m} / z[\mathrm{M}-\mathrm{H}]^{-}$, the MS2 secondary ions were $793 \mathrm{~m} / z\left[\mathrm{M}-\mathrm{H}-\mathrm{glc}^{-}\right.$, the MS3 ions were $613 \mathrm{~m} / z\left[\mathrm{M}-\mathrm{H}-2 \mathrm{glc}-\mathrm{H}_{2} \mathrm{O}\right]^{-}$, and the MS4 ions were $455 \mathrm{~m} / z[\mathrm{M}-\mathrm{H}-2 \mathrm{glc}-\mathrm{glcA}]^{-}$, which were oleanolic acid aglycone. Above all, the major compound in CMG was ginsenoside Ro, which is consistent with a previous study [19]. The structure of that compound is shown in Figure 2b.

\subsection{Effects of CMG on Forelimb Grip Strength}

After four weeks of CMG supplementation, the forelimb grip strength (Table 1, Figure 3) was 1.27-fold higher in the CMG-5X mice than in the vehicle treatment mice $(p<0.0001)$. In the trend analysis, absolute forelimb grip strength dose-dependently increased with increasing CMG dose $(p<0.0001)$.

Table 1. Effect of CMG (Changbai Mountain Ginseng) supplementation for four weeks on exercise performance.

\begin{tabular}{lcccc}
\hline Exercise Performance & Vehicle & CMG-1X & CMG-5X & Trend Analysis \\
\hline Forelimb grip strength $(\mathrm{g})$ & $114 \pm 13^{\mathrm{a}}$ & $125 \pm 10^{\mathrm{a}}$ & $145 \pm 14^{\mathrm{b}}$ & $<0.0001(\uparrow)$ \\
Weight-loaded Swimming time (min) & $2.5 \pm 0.4^{\mathrm{a}}$ & $4.1 \pm 1.4^{\mathrm{b}}$ & $4.0 \pm 1.1^{\mathrm{b}}$ & $0.0017(\uparrow)$ \\
\hline All mice were sacrificed at the end of the experiment and examined for serum levels of clinical biochemistry. \\
Values are mean \pm SD for $n=8$ mice per group. Values in the same line with different superscripts letters \\
(a and b) differ significantly, $p<0.05$ by one-way ANOVA. The arrows up mean dose-dependently increase by \\
CMG supplementation.
\end{tabular}




\subsection{Effect of CMG on Exercise Performance in a Weight-Loaded Swimming Test}

Energy metabolism during muscular activity determines the level of physiological fatigue, and exercise endurance is an important index for evaluating anti-fatigue treatment. Endurance swimming times were $2.5 \pm 0.4,4.1 \pm 1.4$, and $4.0 \pm 1.1 \mathrm{~min}$ with the vehicle, $\mathrm{CMG}-1 \mathrm{X}$, and CMG-5X treatments, respectively (Table 1, Figure 3). The endurance swimming times were respectively 1.67- and 1.60-fold longer in the CMG-1X and CMG-5X groups than in the vehicle treatment group ( $p=0.0055$ and $p=0.0196$, respectively).

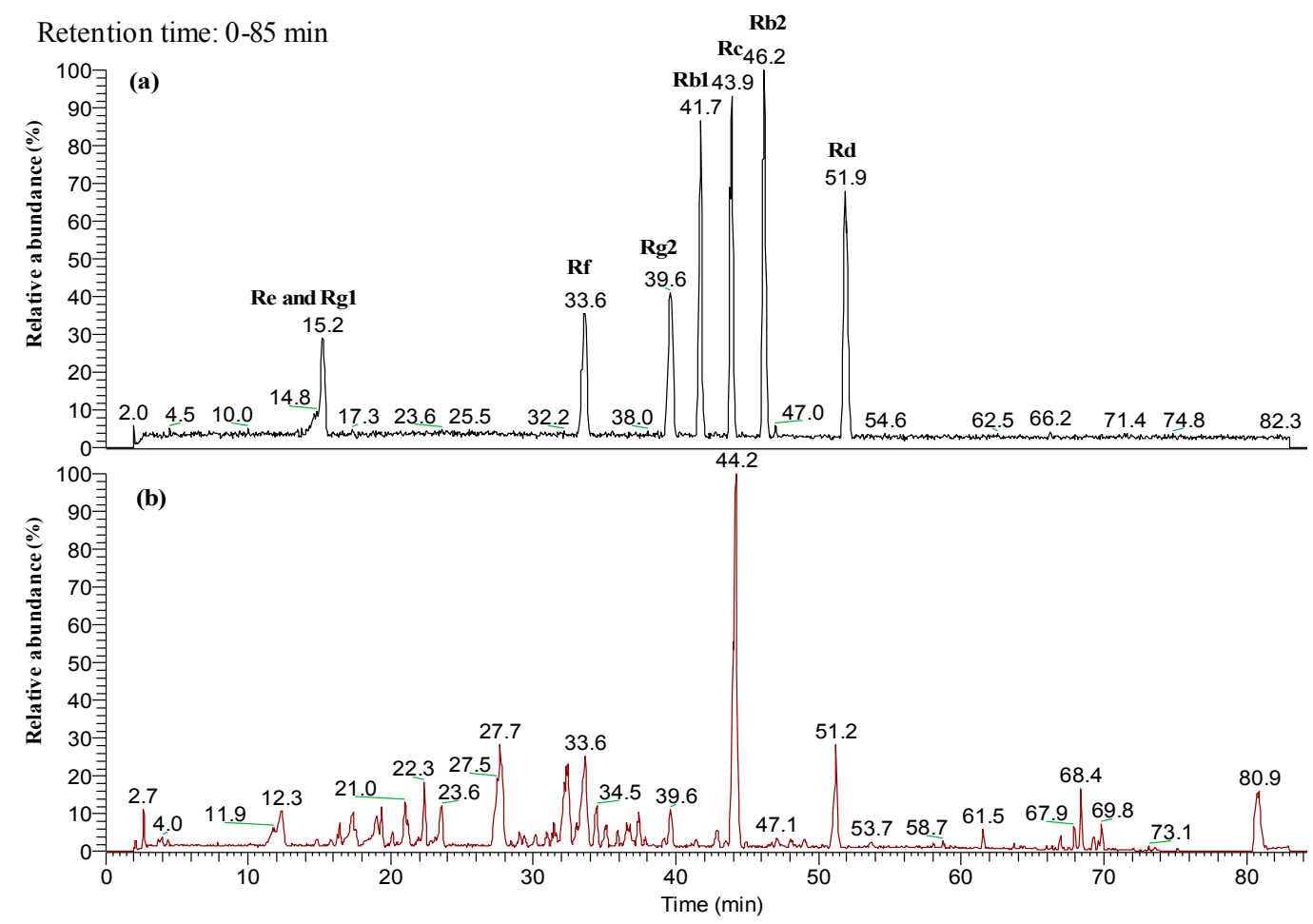

Figure 1. Base peak HPLC-ESI-MS chromatogram of ginsenosides in CMG extract. Ginseng ginsenoside mix standard (a, upper panel); CMG extract (b, bottom panel).

(a)

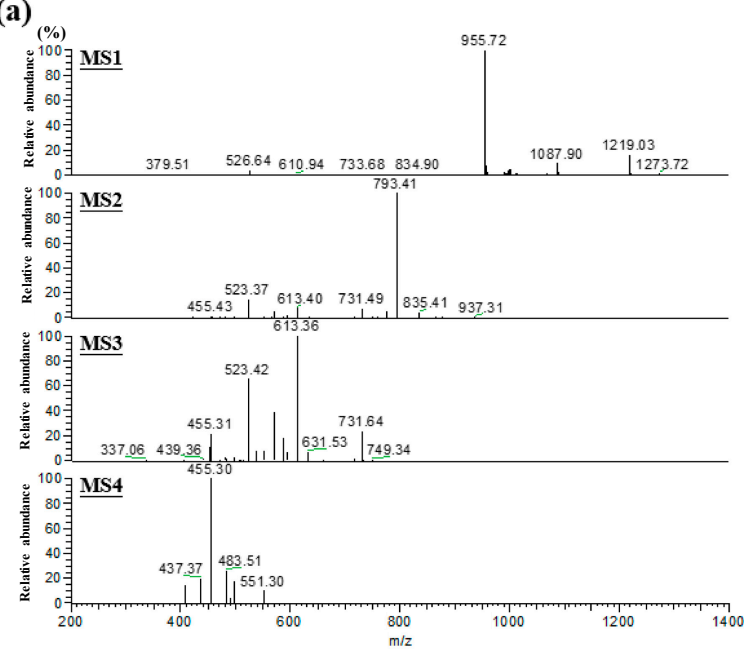

(b)

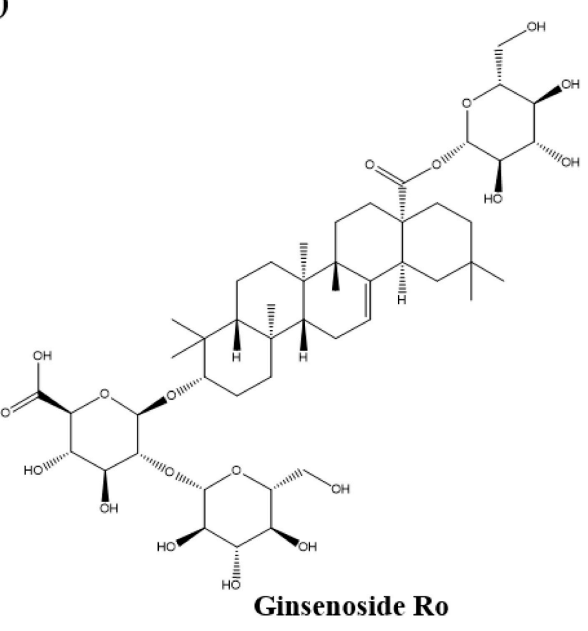

Figure 2. Characterized CMG extract by HPLC-ESI-MS/MSn. HPLC-ESI-MS/MSn chromatogram of major ginsenoside in CMG extract (a); and the chemical structure of the major compound in CMG extract (b). 


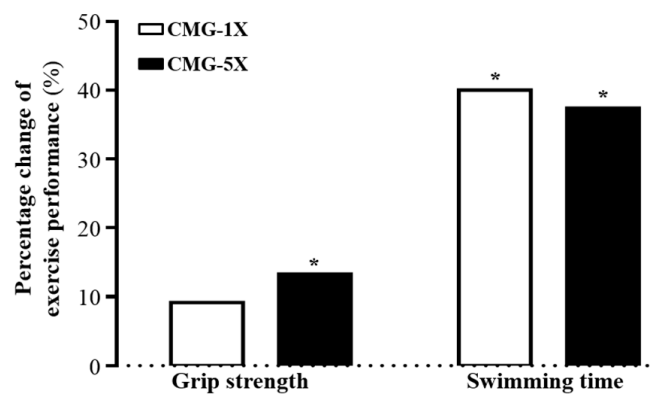

Figure 3. Percentage change with vehicle treatment of CMG (Changbai Mountain Ginseng) supplementation for four weeks on forelimb grip strength and swimming time. Mice were pretreated with vehicle, CMG-1X, or CMG-5X for four weeks before forelimb grip strength was tested. Asterisk $\left.{ }^{*}\right)$, significant difference $(p<0.05)$ from vehicle group.

\subsection{Effect of CMG Supplementation on Serum Lactate, Ammonia, Glucose, CK, and BUN Levels after Acute Exercise Challenge}

In the present study, we determined anti-fatigue related serum levels after 15 min swimming (Table 2, Figure 4). Lactate levels were significantly lower in the CMG-1X and CMG-5X supplementation groups (36.2\%, $p<0.0001$ and $37.0 \%, p<0.005$, respectively) than in the vehicle treatment group, indicating that CMG supplementation was a factor in the removal and utilization of blood lactate after exercise. Serum ammonia levels were respectively $34.9 \%(p=0.0123)$ and $45.9 \%$ $(p=0.0017)$ lower in the CMG-1X and CMG-5X groups than in the vehicle treatment group. The trend analysis revealed that lactate and serum ammonia level dose-dependently decreased with increasing CMG dose $(p<0.0001)$. Thus, continuous supplementation with CMG for four weeks decreased lactate and ammonia levels during exercise. Serum CK level is an important clinical biomarker of muscle damage. Therefore, reduced serum CK levels could represent increased recovery capacity from exercise-induced muscle damage. CK levels were respectively 55.9\% and 56.3\% $(p<0.0001)$ lower in the CMG-1X and CMG-5X groups than in the vehicle group, indicating that CMG supplementation may ameliorate skeletal muscle injury induced by acute exercise challenge.

Blood glucose level is an important index for performance maintenance during exercise. The glucose level was 1.4-fold higher $(p=0.0001)$ in the CMG-5X group than in the vehicle group (Figure 4). The trend analysis showed that serum glucose levels dose-dependently increased with CMG supplementation dose $(p<0.0001)$. BUN is an important biochemical parameter related to fatigue. The BUN level is a measure of the amount of nitrogen in blood from the waste product of urea. Urea serves an important role in the metabolism of nitrogen-containing compounds. Serum BUN levels were respectively $21.4 \%$ and $25.0 \%(p<0.0001)$ lower in the CMG-1X and CMG-5X groups than in the vehicle group. Trend analysis showed that absolute BUN level decreased dose-dependently with increases in CMG dose $(p<0.0001)$. Therefore, CMG may be considered as an ergogenic supplement that facilitates the removal of metabolic waste during exercise.

Table 2. Effect of CMG supplementation on serum levels after acute exercise challenge.

\begin{tabular}{ccccc}
\hline Serum Levels after 15 min Swimming & Vehicle & CMG-1X & CMG-5X & Trend Analysis \\
\hline Lactate $(\mathrm{mmol} / \mathrm{L})$ & $7.3 \pm 0.7^{\mathrm{a}}$ & $4.7 \pm 0.8^{\mathrm{b}}$ & $4.6 \pm 0.4^{\mathrm{b}}$ & $<0.0001(\downarrow)$ \\
$\mathrm{NH}_{3}(\mu \mathrm{mol} / \mathrm{L})$ & $223 \pm 95^{\mathrm{a}}$ & $145 \pm 15^{\mathrm{b}}$ & $121 \pm 20^{\mathrm{b}}$ & $<0.0001(\downarrow)$ \\
$\mathrm{CK}(\mathrm{U} / \mathrm{L})$ & $907 \pm 328^{\mathrm{a}}$ & $400 \pm 75^{\mathrm{b}}$ & $397 \pm 37^{\mathrm{b}}$ & $0.0002(\downarrow)$ \\
Glucose $(\mathrm{mg} / \mathrm{dL})$ & $125 \pm 22^{\mathrm{a}}$ & $143 \pm 19^{\mathrm{b}}$ & $177 \pm 25^{\mathrm{b}}$ & $<0.0001(\uparrow)$ \\
BUN $(\mathrm{mg} / \mathrm{dL})$ & $34.6 \pm 4.5^{\mathrm{a}}$ & $27.2 \pm 1.6^{\mathrm{b}}$ & $25.9 \pm 2.2^{\mathrm{b}}$ & $<0.0001(\downarrow)$ \\
\hline
\end{tabular}

All mice were sacrificed at the end of experiment and examined for serum levels of clinical biochemistry. Values are mean $\pm \mathrm{SD}$ for $n=8$ mice per group. Values in the same line with different superscripts letters ( $\mathrm{a}$ and $\mathrm{b}$ ) differ significantly, $p<0.05$ by one-way ANOVA. CK, creatine kinase; BUN, blood urea nitrogen. The arrows up (down) mean dose-dependently increase (decrease) by CMG supplementation. 


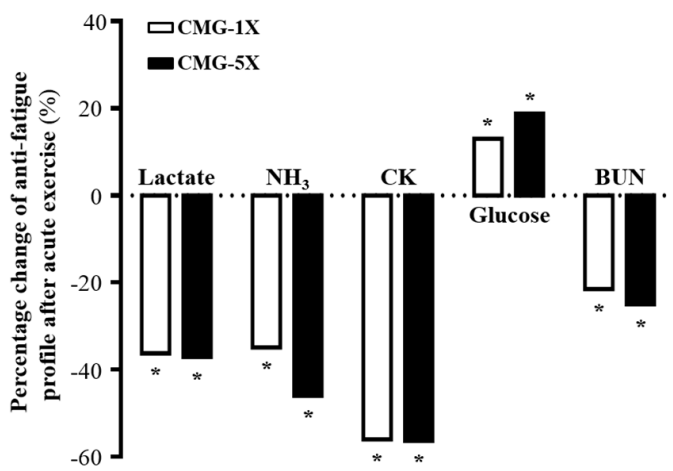

Figure 4. Percentage change with vehicle treatment of CMG supplementation on serum levels of lactate, ammonia, creatine kinase (CK), glucose, and blood urea nitrogen (BUN) after acute exercise challenge. Asterisk $\left(^{*}\right)$, significant difference $(p<0.05)$ from vehicle group.

\subsection{Effect of CMG Supplementation for 4 Weeks on Hepatic and Muscle Glycogen Levels}

Glycogen storage directly affects exercise ability, so we measured the glycogen contents of liver and muscle tissues (Table 3). The liver glycogen content suggested no significant differences with all treatments (Figure 5); however, the liver glycogen was slightly higher in the supplementation groups than in the vehicle group. On the other hand, the muscle glycogen content was 1.38 -fold ( $p=0.0073)$ higher in the CMG-1X group than in the vehicle group. Thus, CMG-1X slightly increased liver and muscle glycogen storage.

Table 3. Effects of CMG supplementation on glycogen levels in liver and muscle.

\begin{tabular}{ccccc}
\hline Glycogen Contents & Vehicle & CMG-1X & CMG-5X & Trend Analysis \\
\hline Liver $(\mu \mathrm{g} / \mathrm{g})$ & $8.0 \pm 4.0$ & $11.3 \pm 6.1$ & $9.2 \pm 2.0$ & 0.3564 \\
Muscle $(\mu \mathrm{g} / \mathrm{g})$ & $0.16 \pm 003^{\mathrm{a}}$ & $0.23 \pm 0.06^{\mathrm{b}}$ & $0.15 \pm 0.03^{\mathrm{a}}$ & 0.4625 \\
\hline
\end{tabular}

All mice were sacrificed at the end of experiment and examined for serum levels of clinical biochemistry. Values are mean $\pm \mathrm{SD}$ for $n=8$ mice per group. Values in the same line with different superscripts letters $(\mathrm{a}, \mathrm{b})$ differ significantly, $p<0.05$ by one-way ANOVA.

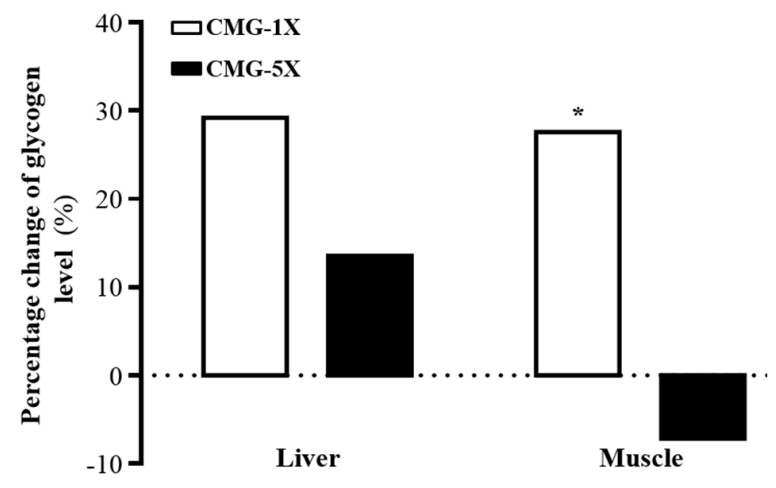

Figure 5. Percentage change with vehicle treatment of CMG supplementation on glycogen levels in liver and muscle. Asterisk $\left(^{*}\right)$, significant difference $(p<0.05)$ from vehicle group.

\subsection{General Characteristics of Mice with CMG Supplementation for Four Weeks}

Initial BW did not differ among the vehicle, CMG-1X, and CMG-5X groups (Table 4). After four-week supplementation with CMG, the final BW was not different in each group. Daily intake of diet and water was not different in the vehicle and CMG supplementation groups, either. Thus, four-week supplementation with CMG affected neither BW nor water and diet intake. We also 
measured the effect of CMG on muscle mass and relative muscle weight (different tissue weights adjusted for individual BW \%). The muscle weights (gastrocnemius and soleus muscles) were, respectively, 1.08- ( $p=0.0021)$ and 1.09-fold ( $p=0.0005)$ higher in the CMG-1X and CMG-5X groups than in the vehicle group. Trend analysis showed that muscle weight dose-dependently increased with CMG supplementation $(p<0.0001)$. The relative muscle weights $(\%)$ were, respectively, $1.17-(p=0.021)$ and 1.23-fold ( $p=0.0032$ ) greater in the CMG-1X and CMG-5X groups than in the vehicle group. Trend analysis also showed significant dose-dependent increases in muscle weight $(p<0.0001)$ and relative muscle weight ( $p=0.0004)$ with CMG supplementation. In addition, the BAT weights were, respectively, 1.18- ( $p=0.006)$ and 1.24-fold ( $p=0.0006)$ higher in the CMG-1X and CMG-5X groups than in the vehicle group. Trend analysis revealed that BAT weight and relative BAT weight (both $p<0.0001$ ) had significant dose-dependent effects with CMG supplementation. Thus, supplementation with CMG for four weeks could be beneficial to muscle mass and BAT weight. According to this data, we found no gross abnormalities attributed to CMG when weighing organs.

Table 4. General characteristics of mice with Changbai Mountain Ginseng (CMG) supplementation.

\begin{tabular}{ccccc}
\hline Characteristic & Vehicle & CMG-1X & CMG-5X & Trend Analysis \\
\hline Initial BW (g) & $37.2 .6 \pm 1.0$ & $37.3 \pm 1.3$ & $37.8 \pm 1.7$ & 0.2231 \\
Final BW (g) & $38.5 \pm 1.5$ & $38.9 \pm 0.9$ & $38.6 \pm 1.3$ & 0.7732 \\
Food intake (g/day) & $7.3 \pm 0.6$ & $7.2 \pm 0.7$ & $7.2 \pm 0.3$ & 0.8104 \\
Water intake (mL/day) & $8.5 \pm 0.6$ & $8.6 \pm 0.5$ & $8.3 \pm 0.3$ & 0.1808 \\
Liver (g) & $2.09 \pm 0.16$ & $2.10 \pm 0.10$ & $2.08 \pm 0.14$ & 08814 \\
Kidney (g) & $0.60 \pm 0.05$ & $0.60 \pm 0.03$ & $0.61 \pm 0.05$ & 0.7797 \\
Heart (g) & $0.26 \pm 0.04$ & $0.27 \pm 0.03$ & $0.27 \pm 0.04$ & 0.4513 \\
Lung (g) & $0.22 \pm 0.02$ & $0.22 \pm 0.01$ & $0.22 \pm 0.02$ & 0.9438 \\
Muscle (g) & $0.37 \pm 0.02^{\mathrm{a}}$ & $0.39 \pm 0.01^{\mathrm{b}}$ & $0.40 \pm 0.01^{\mathrm{b}}$ & $<0.0001(\uparrow)$ \\
EFP (g) & $0.33 \pm 0.15$ & $0.31 \pm 0.01$ & $0.29 \pm 0.01$ & 0.8270 \\
BAT (g) & $0.076 \pm 0.011^{\mathrm{a}}$ & $0.090 \pm 0.006^{\mathrm{b}}$ & $0.095 \pm 0.009 \mathrm{~b}$ & $<0.0001(\uparrow)$ \\
Relative liver weight (\%) & $5.43 \pm 0.32$ & $5.40 \pm 0.30$ & $5.38 \pm 0.22$ & 0.8766 \\
Relative kidney weight (\%) & $1.56 \pm 0.11$ & $1.55 \pm 0.08$ & $1.57 \pm 0.13$ & 0.9078 \\
Relative Heart weight (\%) & $0.67 \pm 0.10$ & $0.70 \pm 0.08$ & $0.69 \pm 0.10$ & 0.4836 \\
Relative Lung weight (\%) & $0.58 \pm 0.05$ & $0.56 \pm 0.03$ & $0.58 \pm 0.05$ & 1.0000 \\
Relative Muscle weight $(\%)$ & $0.95 \pm 0.05^{\mathrm{a}}$ & $1.01 \pm 0.03^{\mathrm{b}}$ & $1.03 \pm 0.05^{\mathrm{b}}$ & $0.0004(\uparrow)$ \\
Relative EFP weight $(\%)$ & $0.85 \pm 0.37$ & $0.79 \pm 0.18$ & $0.76 \pm 0.20$ & 0.8081 \\
Relative BAT weight $(\%)$ & $0.20 \pm 0.03^{\mathrm{a}}$ & $0.23 \pm 0.02^{\mathrm{b}}$ & $0.25 \pm 0.03^{\mathrm{b}}$ & $<0.0001(\uparrow)$ \\
\hline
\end{tabular}

Data are mean $\pm \mathrm{SD}, n=8$ mice/group. Values in the same line with different superscripts letters $(\mathrm{a}, \mathrm{b})$ differ significantly, $p<0.05$ by one-way ANOVA. Food efficiency ratio: body weight (BW) gain (g/day)/food intake (g/day). Muscle mass includes both gastrocnemius and soleus muscles in the lower legs. BAT: brown adipose tissue; EFP: epididymal fat pad. Mice were pretreated with vehicle, CMG-1X, or CMG-5X for 4 weeks. The arrows up mean dose-dependently increase by CMG supplementation.

\subsection{Effect of CMG Supplementation on Biochemical Variables at the End of the Experiment}

We observed beneficial effects of CMG on grip strength, exhaustive exercise challenge, and body composition with four-week CMG supplementation. We further investigated whether four-week CMG treatment affected other biochemical markers in healthy mice (Table 5).

Levels of biochemical indices, including aspartate aminotransferase (AST), alanine aminotransferase (ALT), lactate dehydrogenase (LDH), blood urea nitrogen (BUN), creatinine, uric acid (UA), and total cholesterol (TC), were the same among groups ( $p>0.05$, Table 2 ). Serum CK levels were $26.63 \%(p=0.0455)$ lower with CMG-5X than with vehicle treatment. Serum TP and glucose levels were respectively 1.06- $(p=0.0268)$ and 1.14-fold $(p=0.0145)$ higher with CMG-5X than with vehicle treatment. Serum TG levels were significantly lower with CMG-1X $(p=0.0025)$ and CMG-5X $(p=0.0022)$ than with vehicle treatment. Trend analysis showed that serum CK $(p=0.0045)$ and TG $(p<0.0001)$ levels dose-dependently decreased with CMG extract supplementation; serum TP $(p=0.0017)$ and glucose $(p=0.0030)$ dose-dependently increased with CMG extract supplementation. 
In addition, serum albumin slightly increased with CMG-5X treatment, while the albumin levels were still within the normal range. Therefore, long-term daily supplementation with CMG extract may have the potential to protect tissue and enhance nutrient absorption, with increases in serum TP and glucose level. We also examined the tissue morphology. CMG supplementation for four weeks had no adverse effects on major organs such as the liver, skeletal muscle, heart, kidney, lung, and epididymal fat pad (EFP). Therefore, the dose of CMG extract supplementation used in this study was safe (Figure 6).

Table 5. Biochemical analysis with CMG supplementation at the end of the experiment.

\begin{tabular}{ccccc}
\hline Variables & Vehicle & CMG-1X & CMG-5X & Trend Analysis \\
\hline AST (U/L) & $102 \pm 14$ & $102 \pm 11$ & $101 \pm 11$ & 0.5837 \\
ALT (U/L) & $61 \pm 16$ & $59 \pm 19$ & $60 \pm 22$ & 0.7688 \\
LDH (U/L) & $534 \pm 60$ & $528 \pm 82$ & $537 \pm 76$ & 0.9100 \\
CK (U/L) & $560 \pm 140^{\mathrm{b}}$ & $507 \pm 148^{\mathrm{ab}}$ & $413 \pm 124^{\mathrm{a}}$ & $0.0045(\downarrow)$ \\
TP (g/dL) & $4.9 \pm 0.2^{\mathrm{a}}$ & $5.0 \pm 0.4^{\mathrm{ab}}$ & $5.2 \pm 0.2^{\mathrm{b}}$ & $0.0017(\uparrow)$ \\
Albumin (g/dL) & $3.0 \pm 0.1^{\mathrm{a}}$ & $3.0 \pm 0.1^{\mathrm{a}}$ & $3.2 \pm 0.1^{\mathrm{b}}$ & $<0.0001(\uparrow)$ \\
BUN (mg/dL) & $23.6 \pm 1.8$ & $23.9 \pm 1.6$ & $22.5 \pm 1.7$ & 0.4280 \\
Creatinine (mg/dL) & $0.30 \pm 0.03$ & $0.31 \pm 0.04$ & $0.30 \pm 0.02$ & 0.8319 \\
UA (mg/dL) & $1.9 \pm 0.6$ & $1.7 \pm 0.3$ & $1.7 \pm 0.3$ & 0.5904 \\
TC (mg/dL) & $137 \pm 13$ & $133 \pm 8$ & $131 \pm 8$ & 0.3105 \\
TG (mg/dL) & $153 \pm 15^{\mathrm{b}}$ & $124 \pm 22^{\mathrm{a}}$ & $124 \pm 11^{\mathrm{a}}$ & $<0.0001(\downarrow)$ \\
Glucose (mg/dL) & $148 \pm 18^{\mathrm{a}}$ & $155 \pm 16^{\mathrm{ab}}$ & $168 \pm 10^{\mathrm{b}}$ & $0.0030(\uparrow)$ \\
\hline
\end{tabular}

Data are mean $\pm \mathrm{SD}, n=8$ mice/group. Values in the same line with different superscripts letters $(\mathrm{a}, \mathrm{b})$ differ significantly, $p<0.05$ by one-way ANOVA. AST, aspartate aminotransferase; ALT, alanine aminotransferase; $\mathrm{LDH}$, lactate dehydrogenase; CK, creatine kinase; TP, total protein; BUN, blood urea nitrogen; UA, uric acid; $\mathrm{TC}$, total cholesterol; TG, triacylglycerols. The arrows up (down) mean dose-dependently increase (decrease) by CMG supplementation.

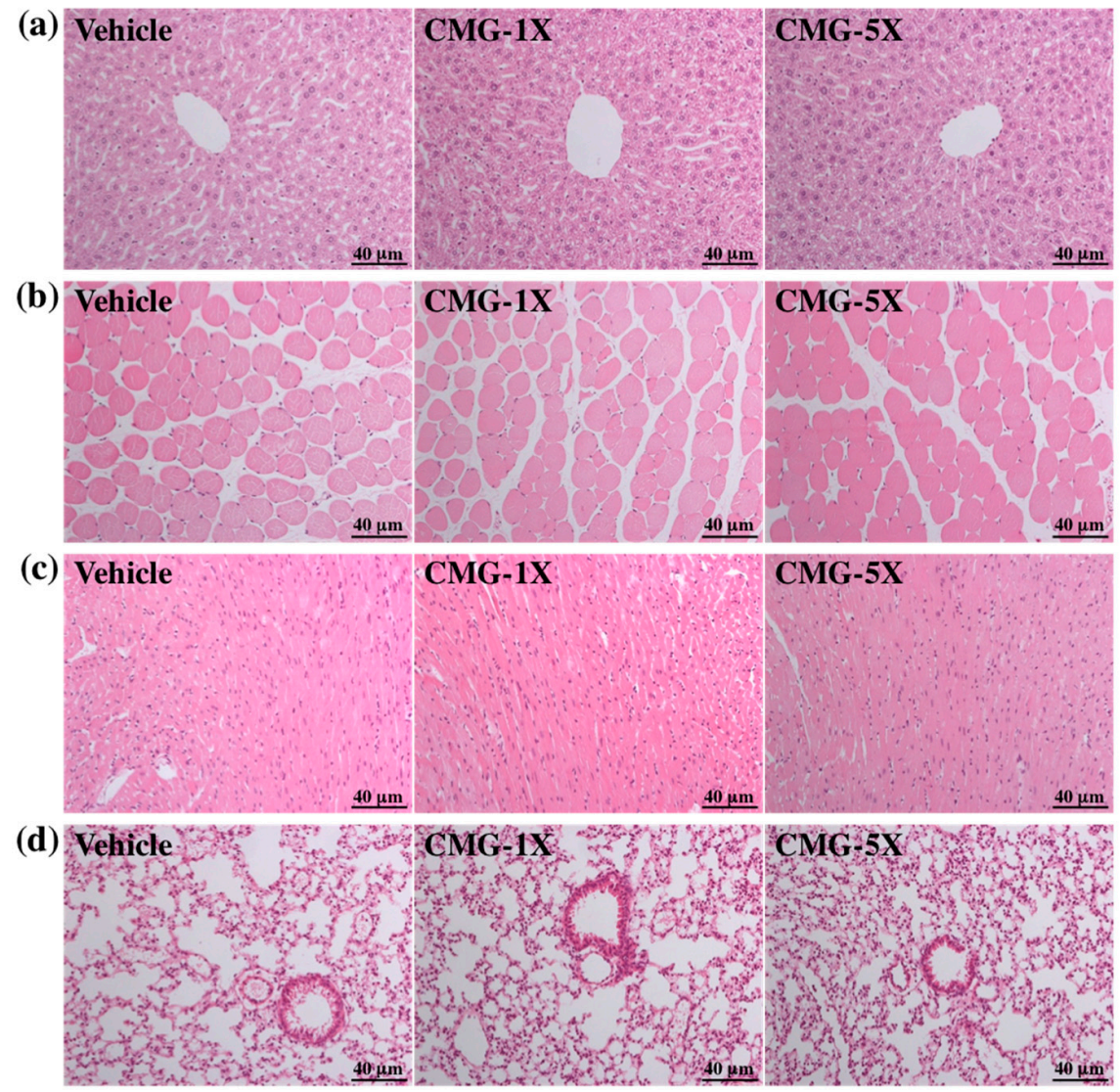

Figure 6. Cont. 


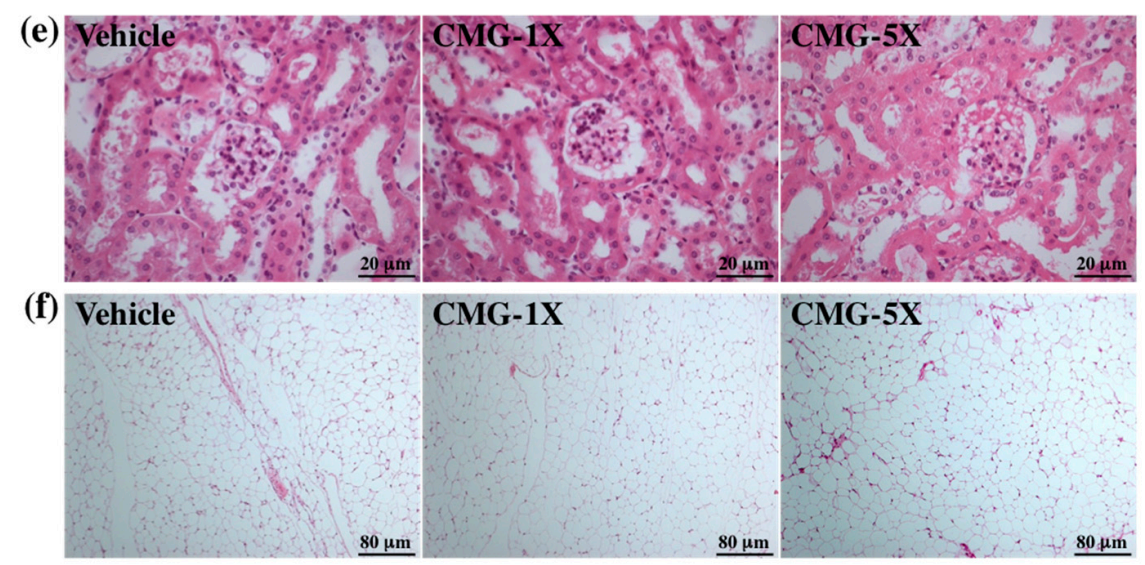

Figure 6. Effect of CMG supplementation on morphology of liver (a); skeletal muscle (b); heart (c); lungs (d); kidney (e); and epididymal fat pad (f). Specimens were photographed by light microscopy. (H and E stain, magnification: (a-d), 200× Scale bar, $40 \mu \mathrm{m} ;(\mathbf{e}), 400 \times$, Scale bar, $20 \mu \mathrm{m} ;(\mathbf{f}), 100 \times$, Scale bar, $80 \mu \mathrm{m})$.

\section{Discussion}

The beneficial effects of ginsenoside species have been well demonstrated in many studies. However, the function of ginsenoside Ro, an oleanane-type saponin, has not been sufficiently investigated. The place of origin and weather could influence ginseng trace elements and secondary metabolites, which could explain why CMG differs from other types of ginseng and has a high content of ginsenoside Ro. In general, programmed exercise training is required to increase grip strength [20]; however, we found that ginsenoside Ro supplementation benefited grip strength even though the test animals underwent no training intervention. Thus, long-term ginsenoside Ro supplementation could benefit the muscle explosive force under non-training conditions. However, few studies have investigated the use of ginseng supplementation to improve muscle strength. According to a previous study, dammarane steroid, a ginseng steroid that is present in many ginseng species, has anti-inflammatory effects on skeletal muscle following a bout of muscle-damaging exercise [21]. Thus, ginsenoside Ro may have an anti-inflammation effect and enhance skeletal muscle performance. Ginseng has a wide range of benefits that could promote physical performance and recovery capacity from interval exercise [22]. We suggest that ginsenoside Ro may improve endurance performance in the absence of training. Further investigation is required to clarify which types of saponins could benefit exercise training for endurance and explosive force performance. In a previous report, 20(R)-ginsenoside Rg3 was demonstrated to increase weight-loaded swimming time [23]. Moreover, ginsenoside Ro may act as a potential ergogenic aid for exercise supplementation.

Exercise-induced muscle fatigue can be evaluated with biochemical indicators, including lactate, ammonia, glucose, CK, and BUN levels [24,25]. The clearance of lactate is thought to reduce peripheral neuromuscular fatigue and have positive effects on muscle function [26]. After acute exercise, relaxation is significantly affected by the blood lactate clearance rate. Approximately $75 \%$ of the total amount of lactate produced is used for oxidative production of energy in the exercising body, and lactate could be utilized for the de novo synthesis of glucose in the liver [27]. During exercise, muscle utilizes glucose from glycogen by intramuscular glycogenolysis and by increased glucose uptake. Regardless, aerobic and resistance exercises increase glucose transporter type 4 (GLUT4) abundance and translocation, thereby increasing serum glucose uptake by a pathway that is not dependent on insulin [28,29]. Ammonia, an important metabolite during energy metabolism for exercise, is generated by different sources. Accumulation of ammonia in the blood and brain during exercise can negatively affect the central nervous system and cause fatigue. The present data suggest that continuous supplementation with CMG extract for four weeks could decrease lactate, ammonia, and BUN accumulation, economize serum glucose levels and, thus, CMG extract could 
have anti-fatigue activity. The effect on CK serum response appears to be related to the magnitude of eccentric contractions involved in the activity and the subsequent extent of muscle disruption [30]. High-intensity exercise challenge can physically or chemically cause tissue damage and muscular cell necrosis [31]. Thus, ginsenoside Ro could reduce muscle damage after exercise.

Concerning glycogen, in our present study, ginsenoside Ro may have influenced hepatic and muscle glycogen metabolism. Previously, it has been reported that ginsenoside suppresses hepatic glucose production through inhibiting the small heterodimer partner (SHP) gene expression [32] or increasing hepatic glycogen storage [33]. Ginseng, or ginsenosides associated with acetyl CoA carboxylase kinase (AMPK) activation, switches off anabolic pathways, including glycolysis, lipolysis, glycogen synthesis, and protein synthesis in the liver [34]. There are at least three mechanisms to regulate the glucose uptake of skeletal muscle. To begin with, glucose delivery to the skeletal muscle cells; then, glucose transport through the cell surface membrane; and, finally, flux through intracellular metabolism [35]. During short-term exercise, the muscle glycogen is the preferred carbohydrate fuel for both aerobic and anaerobic metabolism. When exercise is prolonged, glycogen stores in muscle and liver are depleted during exercise, and blood glucose utilization becomes the main carbohydrate fuel during exercise [36]. Therefore, we suggested that CMG-1X supplementation was the proper dose for recommendation as an ergogenic aid. Our present data also showed that CMG-5X could increase the serum glucose levels by decreasing glycogen storage. We suggested that the ginsenosides may increase glycolysis [37]. During exercise, both serum glucose and muscle glycogen are important fuels. The increase of muscle glucose uptake during exercise depends upon the delivery of glucose (capillary perfusion and plasma glucose concentration) and the permeability of the muscle membrane to glucose [35]. In addition to muscle glycogen content, serum glucose level is the other energy utilization index. Therefore, CMG could modulate the muscle glycogen storage and ginsenoside Ro could be as an ergogenic supplement. It had been reported that ginseng saponin decreases plasma triglyceride levels and inhibits atheroma formation in animals with hypercholesterolemia [38]. The reason why enhanced nutrition absorption with TP was increased by CMG extract supplementation could be that the ginsenosides were biotransformed by intestinal bacteria, which further improved intestinal absorption and bioactivity and diminished the toxicity of the metabolite $[39,40]$.

However, there were still some imitations of this study. The major limitation of the study is that we cannot extrapolate our results to the human because only animal blood of tissue samples were included in this study. Another limitation of the study is the lack of information on the major compound of ginsenoside profiling data from fresh or dried material or during growth of Changbai Mountain Ginseng in different seasons.

\section{Materials and Methods}

\subsection{Preparation of Changbai Mountain Ginseng (CMG) Extract}

CMG specimens were cut into small pieces and soaked in ethanol at ambient temperature for seven days. The extracts were decanted and filtered through Whatman No. 2 filter paper (Sigma, St. Louis, MO, USA), and the filtrates were concentrated in a rotary evaporator before being lyophilized. The yield of CMG was $0.4 \%(1.96 \mathrm{~g} / 470.5 \mathrm{~g} \times 100)$.

\subsection{Liquid Chromatographic-Mass Spectrometry (LC-MS) Analysis of CMG Extract}

One hundred microliters of CMG extract powder $(670 \mathrm{mg} / \mathrm{mL})$ were dissolved in milli-Q water. Before being loaded into SPE (polymeric reversed-phase solid-phase-extraction cartridges; $200 \mathrm{mg} / 3 \mathrm{~mL}$; StrataX, Phenomenex, Denver, CO, USA), the CMG extract liquid was added into $600 \mu \mathrm{L}$ methanol and then eluted out with 10\%-100\% of acetonitrile. The system included an HPLC equipped with a Thermo Finnigan model LXQ linear ion trap mass spectrometer (San Jose, CA, USA) operated in negative ion electrospray mode. A YMC Hydrosphere C18 analytical column $(2.0 \times 150 \mathrm{~mm}, 5 \mu \mathrm{m}$, YMC, Kyoto, Japan) was maintained at room temperature, and a flow-rate of $0.2 \mathrm{~mL} / \mathrm{min}$ was used. 
The mobile phase consisted of water with $0.1 \%$ formic acid (solvent $\mathrm{A}$ ) and acetonitrile with $0.1 \%$ formic acid (solvent B). Gradients were programmed as follows: $21 \%$ B for $0-5 \mathrm{~min}, 21 \%-34 \%$ B for 5-40 min, holding at 34\% B for 40-60 min, 34\%-38\% B for $60-70 \mathrm{~min}, 38 \%-100 \%$ B for $70-80 \mathrm{~min}$, and holding at $100 \%$ B for 80-85 min. A ginseng ginsenosides standard kit (Sigma, G-015, St. Louis, $\mathrm{MO}, \mathrm{USA})$ was prepared for injection volumes of $10 \mu \mathrm{L}$.

\subsection{Animals and Experiment Design}

Male ICR mice (nine weeks old) grown under specific pathogen-free conditions were purchased from BioLASCO (Yi-Lan, Taiwan). All mice were provided a standard laboratory diet (No. 5001; PMI Nutrition International, Brentwood, MO, USA) and distilled water ad libitum, and they were housed with a 12-h light/12-h dark cycle at room temperature $\left(22 \pm 1{ }^{\circ} \mathrm{C}\right)$ and $50 \%-60 \%$ humidity. The Institutional Animal Care and Use Committee (IACUC) of National Taiwan Sport University (NTSU) inspected all animal experiments, and this study conformed to the guidelines of protocol IACUC-105,020 approved by the IACUC ethics committee. In this study, the dose of CMG for humans was $24.4 \mathrm{mg}$ per day (CMG extract). The mice dose (5 mg/ $\mathrm{kg}$ ) we used was converted from a human-equivalent dose (HED) based on body surface area by the following formula from the US Food and Drug Administration: assuming a human weight of $60 \mathrm{~kg}$, the HED for $24.4(\mathrm{mg}) / 60(\mathrm{~kg})=0.406 \times 12.3=5 \mathrm{mg} / \mathrm{kg}$; the conversion coefficient 12.3 was used to account for differences in body surface area between mice and humans as recently described [41]. In total, 24 mice were randomly assigned to three groups (eight mice/group) for daily oral CMG treatment for 4 weeks. The groups and treatment courses were as follows: vehicle; $5 \mathrm{mg} / \mathrm{kg}$ (CMG-1X); and $25 \mathrm{mg} / \mathrm{kg}$ (CMG-5X). The vehicle group received the same volume of solution equivalent to individual body weight (BW). Mice were randomly housed in groups of four per cage.

\subsection{Forelimb Grip Strength Test}

A low-force testing system (Model-RX-5, Aikoh Engineering, Nagoya, Japan) was used to measure the forelimb grip strength of treated mice as previously described [42]. Forelimb grip strength was tested one hour after administration of the indicated CMG supplementation for four weeks. The forelimb grip strengths of all mice were measured on the same day.

\subsection{Swimming Exercise Performance Test}

The swimming endurance test was conducted after the forearm grip strength test for all mice. For the swim-to-exhaustion test, loads corresponding to $5 \%$ of the mouse BW were attached to the tail to evaluate endurance time [43]. The swimming endurance time of each mouse was recorded from the beginning to exhaustion, determined by observing loss of coordinated movements and failure to return to the surface within $7 \mathrm{~s}$. The test of swimming endurance time was performed one hour after administration of the indicated CMG supplementation for 28 days. The swimming exercise performance tests of all mice were performed on the same day as the forelimb grip strength measurement.

\subsection{Determination of Fatigue-Associated Biochemical Variables}

The effect of CMG supplementation on fatigue-associated biochemical indices was evaluated after exercise as previously described [43]. The 15-min swimming test was performed one day after the forelimb grip strength and the swimming exercise performance test. After CMG supplementation for one hour, all mice underwent a 15-min swimming test without weight loading. Immediately after the 15-min test, blood samples were immediately collected from the submandibular duct of mice and centrifuged at $1500 \times g$ and $4^{\circ} \mathrm{C}$ for $10 \mathrm{~min}$ for serum preparation. Serum lactate, ammonia, glucose, creatine kinase $(\mathrm{CK})$, and blood urea nitrogen (BUN) levels were determined with an autoanalyzer (Hitachi 7060, Hitachi, Tokyo, Japan). 


\subsection{Clinical Biochemical Profiles}

Two days after the 15-min swimming test, all mice were sacrificed with $95 \% \mathrm{CO}_{2}$ asphyxiation, and their blood was immediately collected. Blood collected by cardiac puncture was centrifuged at $1500 \times \mathrm{g}$ for $10 \mathrm{~min}$ at $4{ }^{\circ} \mathrm{C}$. Serum was separated by centrifugation and the levels of clinical biochemical variables (CK, albumin, total protein (TP), BUN, creatinine, uric acid (UA), total cholesterol (TC), triacylglycerols (TG), and glucose) were measured with an autoanalyzer (Hitachi 7060, Hitachi, Tokyo, Japan).

\subsection{Histology of Tissues}

Liver, skeletal muscle, heart, lung, kidney, and epididymal fat pad (EFP) tissues were carefully removed, minced, and fixed in $10 \%$ formalin. Samples were embedded in paraffin and cut into $4-\mu \mathrm{m}$ thick slices for morphological and pathological evaluations. Tissues were stained with hematoxylin and eosin ( $\mathrm{H}$ and $\mathrm{E})$ and examined under a light microscope equipped with a CCD camera (BX-51, Olympus, Tokyo) by a veterinary pathologist.

\subsection{Tissue Glycogen Determination and Visceral Organ Weight}

The stored form of glucose is glycogen, which mostly exists in liver and muscle tissue. Liver and muscle tissues were excised after the mice were sacrificed and weighed for glycogen content analysis as described previously [44]. The weights of the liver, skeletal muscle, heart, lung, kidney, epididymal fat pad (EFP), and brown adipose tissue (BAT) related to visceral organs were recorded.

\subsection{Statistical Analysis}

All data are expressed as mean $\pm \mathrm{SD}, n=8$ mice per group. Statistical differences among groups were analyzed with one-way analysis of variance (ANOVA and the Cochran-Armitage test for dose-effect trend analysis with SAS 9.0 (SAS Inst., Cary, NC, USA). $p<0.05$ was considered statistically significant. Differences between groups were analyzed by one-way analysis of variance (ANOVA) using Duncan's post-hoc test, and $p$ values $<0.05$ were considered significant.

\section{Conclusions}

We demonstrated that the major compound of CMG is ginsenoside Ro. CMG supplementation for four weeks significantly improved forelimb grip strength and swimming time to exhaustion in test animals (Figure A1). CMG has anti-fatigue activity; it decreased plasma lactate, ammonia, and CK and increased serum glucose levels, thereby enhancing exercise performance in mice. Exercise-induced fatigue-related parameters including lactate, ammonia, CK, and glucose levels were dose-dependently modulated by CMG supplementation. CMG also has positive effects on the lipid profile, tissue protection, and nutritional status in vivo. In this study, we found that CMG supplementation could increase exercise performance, and increase muscle mass. These findings suggest potential biological mechanisms by which ginsenoside Ro may increase energy utilization via glycolysis and thereby increase serum glucose levels or decrease physical fatigue during exercise. Ginsenoside Ro may be associated with AMPK activation, which regulates metabolic processes, including glycolysis or glycogen synthesis. Previous studies have been demonstrated that ginsenoside has an anti-fatigue effect which related to improve energy metabolism and decrease the oxidative stress of skeletal muscle $[45,46]$. The underlying mechanism might be associated with an increase in the content of adenosine triphosphate (ATP) and an enhancement in the activity of energy metabolic enzymes, but the feasibility of other mechanisms still warrants further study. To our best knowledge, this is the first study to investigated that the ginsenoside Ro-rich CMG exhibits anti-fatigue function. Although we have evidence that revealst CMG could improve exercise performance, there is a lack of studies of ginsenoside-Ro in humans. Therefore, these results should be conducted to verify the biological effectiveness in human studies. 
Acknowledgments: This study was not supported by any grant, outside sources of funding, or industry sponsorship. The authors are grateful to Mon-Chien Lee and Chien-Chao Chiu and Yu-Tang Tung for technical assistance in animal experiments and preparation of the CMG extract. The HPLC analysis was performed by the Research Center for Industry of Human Ecology and the Food Safety Testing Laboratory, College of Human Ecology, Chang Gung University of Science and Technology. We also thank Yu-Ping Liao (Centre for International Affairs, National Taiwan Sport University, Taoyuan City, Taiwan) and John P. Ring (Academic Writing Education Center, National Taiwan University, Taipei City, Taiwan) for their careful reading of the manuscript.

Author Contributions: Chi-Chang Huang and Guo-Dong Ma conceived and designed the experiments; Yi-Ming Chen, Chun-Hui Chiu, Yi-Ju Hsu, Chien-Wen Hou performed the experiments; Yi-Ming Chen, Yi-Ju Hsu, Chun-Hui Chiu and Chi-Chang Huang analyzed the data; Guo-Dong Ma and Chien-Wen Hou contributed reagents and materials; Yi-Ming Chen and Chi-Chang Huang wrote and revised the paper.

Conflicts of Interest: The authors declare no conflict of interest.

\section{Appendix A}

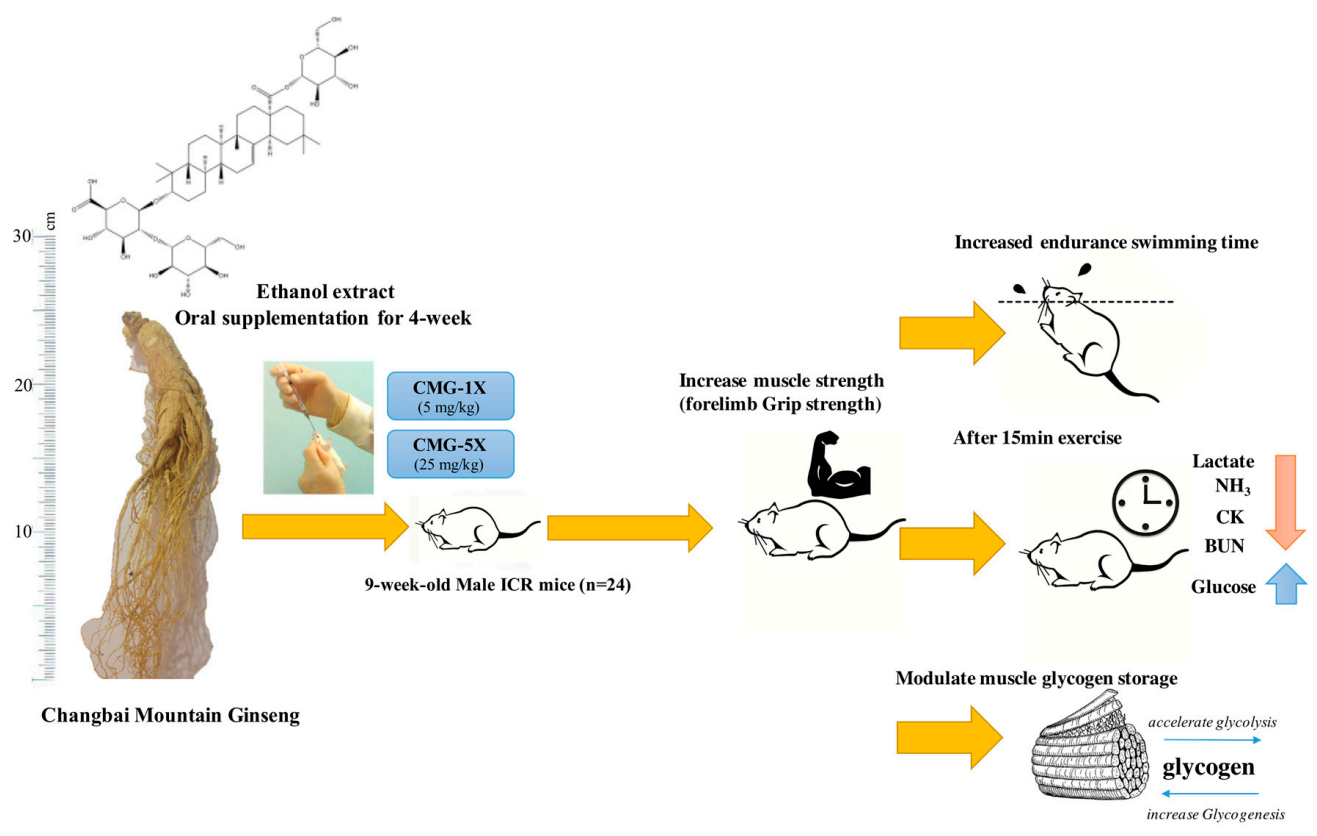

Figure A1. The Bioactivities of Changbai Mountain Ginseng (CMG) Extract on Anti-Fatigue In Vivo.

\section{References}

1. Shuna, L.; Zhaohuai, J. Development Models of Resource-dependent Cities' Transformations and Its Experience and Lessons-Take Baishan City's Development of Transformations as an Example. Energy Procedia 2011, 5, 1626-1630. [CrossRef]

2. Attele, A.S.; Wu, J.A.; Yuan, C.S. Ginseng pharmacology: Multiple constituents and multiple actions. Biochem. Pharmacol. 1999, 58, 1685-1693. [CrossRef]

3. Cui, J. Identification and quantification of ginsenosides in various commercial ginseng preparations. Eur. J. Pharm. Sci. 1995, 3, 77-85. [CrossRef]

4. Tadano, T.; Nakagawasai, O.; Niijima, F. The effects of traditional tonics on fatigue in mice differ from those of the antidepressant imipramine: A pharmacological and behavioral study. Am. J. Chin. Med. 2000, 28, 97-104. [CrossRef] [PubMed]

5. Barton, D.L.; Liu, H.; Dakhil, S.R.; Linquist, B.; Sloan, J.A.; Nichols, C.R.; Loprinzi, C.L. Wisconsin Ginseng (Panax quinquefolius) to improve cancer-related fatigue: A randomized, double-blind trial. J. Natl. Cancer Inst. 2013, 105, 1230-1238. [CrossRef] [PubMed]

6. Bae, H.J.; Chung, S.I.; Lee, S.C.; Kang, M.Y. Influence of Aging Process on the Bioactive Components and Antioxidant Activity of Ginseng (Panax ginseng L.). J. Food Sci. 2014, 79, H2127-H2131. [CrossRef] [PubMed]

7. Hou, Y.L.; Tsai, Y.H.; Lin, Y.H.; Chao, J.C. Ginseng extract and ginsenoside Rb1 attenuate carbon tetrachloride-induced liver fibrosis in rats. BMC Complement. Altern. Med. 2014, 14, 415. [CrossRef] [PubMed] 
8. Lee, S.H.; Oh, M.; Park, J.; Jang, S.Y.; Cheong, S.H.; Lee, H.; Moon, S.H. Antioxidant and anti-inflammatory activities of the ethanolic extract of fermented red ginseng marc. Food Sci. Biotechnol. 2015, 24, 651-657. [CrossRef]

9. Wang, J.R.; Yau, L.F.; Tong, T.T.; Feng, Q.T.; Bai, L.P.; Ma, J.; Jiang, Z.H. Characterization of Oxygenated Metabolites of Ginsenoside Rb1 in Plasma and Urine of Rat. J. Agric. Food Chem. 2015, 63, 2689-2700. [CrossRef] [PubMed]

10. Zhou, Y.; Li, H.Q.; Lu, L.; Fu, D.L.; Liu, A.J.; Li, J.H.; Zheng, G.Q. Ginsenoside Rg1 provides neuroprotection against blood brain barrier disruption and neurological injury in a rat model of cerebral ischemia/reperfusion through downregulation of aquaporin 4 expression. Phytomedicine 2014, 21, 998-1003. [CrossRef] [PubMed]

11. Sun, S.; Qi, L.W.; Du, G.J.; Mehendale, S.R.; Wang, C.Z.; Yuan, C.S. Red notoginseng: Higher ginsenoside content and stronger anticancer potential than Asian and American ginseng. Food Chem. 2011, 125, 1299-1305. [CrossRef] [PubMed]

12. Wang, Y.; Liu, Y.; Zhang, X.Y.; Xu, L.H.; Ouyang, D.Y.; Liu, K.P.; He, X.H. Ginsenoside Rg1 regulates innate immune responses in macrophages through differentially modulating the NF- $\mathrm{KB}$ and PI3K/Akt/mTOR pathways. Int. Immunopharmacol. 2014, 23, 77-84. [CrossRef] [PubMed]

13. Liu, Z.; Li, W.; Li, X.; Zhang, M.; Chen, L.; Zheng, Y.N.; Ruan, C.C. Antidiabetic effects of malonyl ginsenosides from Panax ginseng on type 2 diabetic rats induced by high-fat diet and streptozotocin. J. Ethnopharmacol. 2013, 145, 233-240. [CrossRef] [PubMed]

14. Lee, C.H.; Kim, J.H. A review on the medicinal potentials of ginseng and ginsenosides on cardiovascular diseases. J. Ginseng Res. 2014, 38, 161-166. [CrossRef] [PubMed]

15. Lim, W.; Mudge, K.W.; Vermeylen, F. Effects of population, age, and cultivation methods on ginsenoside content of wild American ginseng (Panax quinquefolium). J. Agric. Food Chem. 2005, 53, 8498-8505. [CrossRef] [PubMed]

16. Wang, J.; Li, S.; Fan, Y.; Chen, Y.; Liu, D.; Cheng, H.; Zhou, Y. Anti-fatigue activity of the water-soluble polysaccharides isolated from Panax ginseng C.A. Meyer. J. Ethnopharmacol. 2010, 130, 421-423. [CrossRef] [PubMed]

17. Wu, R.E.; Huang, W.C.; Liao, C.C.; Chang, Y.K.; Kan, N.W.; Huang, C.C. Resveratrol protects against physical fatigue and improves exercise performance in mice. Molecules 2013, 18, 4689-4702. [CrossRef] [PubMed]

18. Hsu, Y.J.; Chiu, C.C.; Li, Y.P.; Huang, W.C.; Huang, Y.T.; Huang, C.C.; Chuang, H.L. Effect of intestinal microbiota on exercise performance in mice. J. Strength Cond. Res. 2015, 29, 552-558. [CrossRef] [PubMed]

19. Wang, H.P.; Zhang, Y.B.; Yang, X.W.; Yang, X.B.; Xu, W.; Xu, F.; Zhang, L.X. High-Performance Liquid Chromatography with Diode Array Detector and Electrospray Ionization Ion Trap Time-of-Flight Tandem Mass Spectrometry to Evaluate Ginseng Roots and Rhizomes from Different Regions. Molecules 2016, 21, 603. [CrossRef] [PubMed]

20. Chen, W.C.; Huang, W.C.; Chiu, C.C.; Chang, Y.K.; Huang, C.C. Whey protein improves exercise performance and biochemical profiles in trained mice. Med. Sci. Sports Exerc. 2014, 46, 1517-1524. [CrossRef] [PubMed]

21. Yu, S.H.; Huang, C.Y.; Lee, S.D.; Hsu, M.F.; Wang, R.Y.; Kao, C.L.; Kuo, C.H. Decreased eccentric exercise-induced macrophage infiltration in skeletal muscle after supplementation with a class of ginseng-derived steroids. PLoS ONE 2014, 9, e114649. [CrossRef] [PubMed]

22. Engels, H.J.; Fahlman, M.M.; Wirth, J.C. Effects of ginseng on secretory IgA, performance, and recovery from interval exercise. Med. Sci. Sports Exerc. 2003, 35, 690-696. [CrossRef] [PubMed]

23. Tang, W.; Zhang, Y.; Gao, J.; Ding, X.; Gao, S. The anti-fatigue effect of 20(R)-ginsenoside Rg3 in mice by intranasally administration. Biol. Pharm. Bull. 2008, 31, 2024-2027. [CrossRef] [PubMed]

24. Strojnik, V.; Komi, P.V. Fatigue after submaximal intensive stretch-shortening cycle exercise. Med. Sci. Sports Exerc. 2000, 32, 1314-1319. [CrossRef] [PubMed]

25. Izquierdo, M.; González-Izal, M.; Navarro-Amezqueta, I.; Calbet, J.A.; Ibañez, J.; Malanda, A.; Mallor, F.; Häkkinen, K.; Kraemer, W.J.; Gorostiaga, E.M. Effects of strength training on muscle fatigue mapping from surface EMG and blood metabolites. Med. Sci. Sports Exerc. 2011, 43, 303-311. [CrossRef] [PubMed]

26. White, G.E.; Wells, G.D. The effect of on-hill active recovery performed between runs on blood lactate concentration and fatigue in alpine ski racers. J. Strength Cond. Res. 2015, 29, 800-806. [CrossRef] [PubMed]

27. Brooks, S.P.; Storey, K.B. A quantitative evaluation of the effect of enzyme complexes on the glycolytic rate in vivo: Mathematical modeling of the glycolytic complex. J. Theor. Biol. 1991, 149, 361-375. [CrossRef] 
28. Colberg, S.R.; Sigal, R.J.; Fernhall, B.; Regensteiner, J.G.; Blissmer, B.J.; Rubin, R.R.; Braun, B. Exercise and type 2 diabetes the American College of Sports Medicine and the American Diabetes Association: Joint position statement executive summary. Diabetes Care 2010, 33, 2692-2696. [CrossRef] [PubMed]

29. Adams, O.P. The impact of brief high-intensity exercise on blood glucose levels. Diabetes Metab. Syndr. Obes. 2013, 6, 113-122. [CrossRef] [PubMed]

30. Baird, M.F.; Graham, S.M.; Baker, J.S.; Bickerstaff, G.F. Creatine-kinase-and exercise-related muscle damage implications for muscle performance and recovery. J. Nutr. Metab. 2012, 2012, 960363. [CrossRef] [PubMed]

31. Coyle, E.F.; Hagberg, J.M.; Hurley, B.F.; Martin, W.H.; Ehsani, A.A.; Holloszy, J.O. Carbohydrate feeding during prolonged strenuous exercise can delay fatigue. J. Appl. Physiol. Respir. Environ. Exerc. Physiol. 1983, 55, 230-235. [PubMed]

32. Yuan, H.D.; Quan, H.Y.; Kim, S.J.; Jung, M.S.; Chung, S.H. Ginsenoside Rg2 induces orphan nuclear receptor SHP gene expression and inactivates GSK3 $\beta$ via AMP-activated protein kinase to inhibit hepatic glucose production in HepG2 cells. Chem. Biol. Interact. 2012, 195, 35-42. [CrossRef] [PubMed]

33. Jeong, K.J.; Kim, G.W.; Chung, S.H. AMP-activated protein kinase: An emerging target for ginseng. J. Ginseng Res. 2014, 38, 83-88. [CrossRef] [PubMed]

34. Im Chung, S.; Nam, S.J.; Xu, M.; Kang, M.Y.; Lee, S.C. Aged ginseng (Panax ginseng Meyer) reduces blood glucose levels and improves lipid metabolism in high fat diet-fed mice. Food Sci. Biotechnol. 2016, 25, 267-273. [CrossRef]

35. Jensen, T.E.; Richter, E.A. Regulation of glucose and glycogen metabolism during and after exercise. J. Physiol. 2012, 590, 1069-1076. [CrossRef] [PubMed]

36. Coggan, A.R.; Coyle, E.F. Carbohydrate Ingestion during Prolonged Exercise: Effects on Metabolism and Performance. Exerc. Sport Sci. Rev. 1991, 19, 1-40. [CrossRef] [PubMed]

37. Radad, K.; Gille, G.; Moldzio, R.; Saito, H.; Rausch, W.D. Ginsenosides Rb1 and Rg1 effects on mesencephalic dopaminergic cells stressed with glutamate. Brain Res. 2004, 1021, 41-53. [CrossRef] [PubMed]

38. Lee, L.S.; Cho, C.W.; Hong, H.D.; Lee, Y.C.; Choi, U.K.; Kim, Y.C. Hypolipidemic and antioxidant properties of phenolic compound-rich extracts from white ginseng (Panax ginseng) in cholesterol-fed rabbits. Molecules 2013, 18, 12548-12560. [CrossRef] [PubMed]

39. Li, H.; Zhou, M.; Zhao, A.; Jia, W. Traditional Chinese medicine: Balancing the gut ecosystem. Phytother. Res. 2009, 23, 1332-1335. [CrossRef] [PubMed]

40. Wang, H.Y.; Qi, L.W.; Wang, C.Z.; Li, P. Bioactivity enhancement of herbal supplements by intestinal microbiota focusing on ginsenosides. Am. J. Chin. Med. 2011, 39, 1103-1115. [CrossRef] [PubMed]

41. Chen, Y.M.; Lin, C.L.; Wei, L.; Hsu, Y.J.; Chen, K.N.; Huang, C.C.; Kao, C.H. Sake Protein Supplementation Affects Exercise Performance and Biochemical Profiles in Power-Exercise-Trained Mice. Nutrients 2016, 8 , 106. [CrossRef] [PubMed]

42. Chen, Y.M.; Tsai, Y.H.; Tsai, T.Y.; Chiu, Y.S.; Wei, L.; Chen, W.C.; Huang, C.C. Fucoidan supplementation improves exercise performance and exhibits anti-fatigue action in mice. Nutrients 2014, 7, 239-252. [CrossRef] [PubMed]

43. Huang, W.C.; Chiu, W.C.; Chuang, H.L.; Tang, D.W.; Lee, Z.M.; Wei, L.; Huang, C.C. Effect of curcumin supplementation on physiological fatigue and physical performance in mice. Nutrients 2015, 7, 905-921. [CrossRef] [PubMed]

44. Huang, W.C.; Lin, C.I.; Chiu, C.C.; Lin, Y.T.; Huang, W.K.; Huang, H.Y.; Huang, C.C. Chicken essence improves exercise performance and ameliorates physical fatigue. Nutrients 2014, 6, 2681-2696. [CrossRef] [PubMed]

45. Tan, S.J.; Li, N.; Zhou, F.; Dong, Q.T.; Zhang, X.D.; Chen, B.C.; Yu, Z. Ginsenoside Rb1 improves energy metabolism in the skeletal muscle of an animal model of postoperative fatigue syndrome. J. Surg. Res. 2014, 191, 344-349. [CrossRef] [PubMed]

46. Zhuang, C.L.; Mao, X.Y.; Liu, S.; Chen, W.Z.; Huang, D.D.; Zhang, C.J.; Yu, Z. Ginsenoside Rb1 improves postoperative fatigue syndrome by reducing skeletal muscle oxidative stress through activation of the PI3K/Akt/Nrf2 pathway in aged rats. Eur. J. Pharmacol. 2014, 740, 480-487. [CrossRef] [PubMed]

Sample Availability: Not available.

(C) 2017 by the authors; licensee MDPI, Basel, Switzerland. This article is an open access article distributed under the terms and conditions of the Creative Commons Attribution (CC BY) license (http:/ / creativecommons.org/licenses/by/4.0/). 